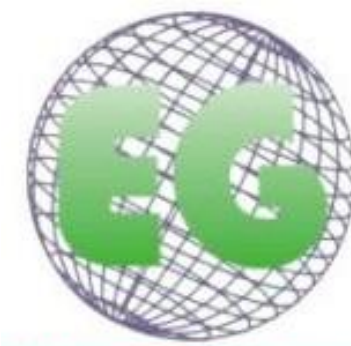

ISSN 1695-6141 $N^{\circ} 48$

\title{
Estrategias Maternas de Alimentación y Actividad Física para prevenir la Diabetes Mellitus Tipo II en Hijos Escolares
}

Maternal Nutrition and Physical Activities Strategies to Prevent Type II Diabetes Mellitus in School Children

JM Santos-Flores ${ }^{1}$

MC Guevara-Valtier ${ }^{2}$

MA Vega-Grimaldo ${ }^{1}$

JM Gutiérrez.Valverde ${ }^{3}$

MA Paz-Morales ${ }^{2}$

${ }^{1}$ Licenciado en Enfermería. Universidad Autónoma de Nueva León. México.

2 Doctorado en Educación. Universidad Autónoma de Nueva León. México.

${ }^{3}$ Doctorado en Ciencias de Enfermería. Universidad Autónoma de Nueva León. México.

E-mail: santos meme10@hotmail.com

http://dx.doi.org/10.6018/eglobal.16.4.256751

Recibido: $17 / 04 / 2016$

Aceptado: 08/08/2016

\section{RESUMEN:}

Introducción: La Diabetes Mellitus Tipo 2 constituye un problema de salud pública importante, es una de las enfermedades crónicas más frecuentes, causa disminución de la calidad de vida, es por ello que es necesario conocer las estrategias de alimentación y actividad física para su prevención desde etapas tempranas de la vida.

Objetivo: Conocer las estrategias de alimentación y actividad física que realizan las madres para prevenir la Diabetes Mellitus Tipo 2 en hijos escolares.

Materiales y métodos: Prueba piloto de un estudio descriptivo con un muestreo aleatorio simple en 30 madres con hijos escolares realizado en una escuela primaria ubicada en el municipio de Sabinas Hidalgo Nuevo León, México. Fue aplicado el instrumento "Escala de Estrategias Parentales de Alimentación y Actividad Física" de 26 ítems.

Resultados: Se encontró que la media más alta fue de Reforzamiento con una $\tilde{x}$ de 64.00 (DE= 18.84) la cual está dirigida a elogiar a los hijos cuando realizan conductas saludables y más baja Monitoreo con $51.33(\mathrm{DE}=20.80)$ que se define como el grado en el cual los padres supervisan las conductas saludables de sus hijos.

Conclusión: Los niños al ejercer conductas saludables, las madres elogian y tienen un mayor control sobre ellos en favor de que realicen lo mayormente deseable en su actividad física y si estos, al contrario realizan conductas no saludables respecto a su alimentación se les limita, supervisa y sancionan tales conductas.

Palabras clave: Estrategias; crianza del niño; madres; diabetes mellitus. 


\begin{abstract}
:
Introduction: Type 2 Diabetes Mellitus is a major public health problem, it is one of the most frequent chronic diseases and causes a decrease in quality of life, and therefore, it is necessary to know the major strategies of nutrition and physical activity for prevention since the early stages of life.

Objective: To know the strategies of nutrition and physical activity performed by mothers to prevent Type 2 Diabetes Mellitus in school children.

Materials and methods Pilot test of a descriptive study with a simple random sampling in 30 mothers with school children carried out in an elementary school located in the community of Sabinas Hidalgo, Nuevo León, Mexico. The instrument "Scale of Parental Strategies for Nutrition and Physical Activity" of 26 items was applied. Results: It was found that the highest measure was of Reinforcement with a $\tilde{x}$ of 64.00 ( $\mathrm{SE}=18.84$ ) which is aimed to praising children when they perform healthy behaviors and a lower Supervision with 51.33 (SE =20.80) defined as the degree in which parents supervise their children's healthy behaviors.

Conclusion: Children, when exercising healthy behaviors, mothers praise them and have a greater control over them in favor of doing the most desirable in their physical activity and if they, in contrast, perform unhealthy behaviors regarding to their nutrition will be limited, supervised and sanctioned such conducts.
\end{abstract}

Keywords: Strategies; Child rearing; mothers; diabetes mellitus.

\title{
INTRODUCCIÓN
}

La diabetes mellitus tipo 2 constituye un problema en salud redundante porque es una de las enfermedades crónicas más frecuentes, causa varias complicaciones agudas (cetoacidosis, hiperglicemia, hipoglucemia) y crónicas (retinopatía, nefropatía, neuropatía, enfermedad isquémica del corazón) disminuyendo la calidad de vida ${ }^{(1)}$.

De acuerdo con la Encuesta Nacional de Salud y Nutrición ${ }^{(1)}$, hay cerca de 6.5 millones de personas que refieren haber sido diagnosticadas con diabetes, y según el Instituto Nacional de Estadística y Geografía ${ }^{(2)}$ en menores de 10 años hay una incidencia de 3.83 por cada 100 mil habitantes y de 10 a 14 años es de 10.85 por cada 100 mil habitantes.

Estas evidencias confirman que la Diabetes Mellitus Tipo 2 (DM2) no es exclusiva de los adultos, sino que también afecta a la población infantil, se conoce que entre los factores de riesgo para la DM2 se encuentran la historia familiar de la enfermedad, alto o bajo peso al nacer; sin embargo, los factores de riesgo más prevalentes son el sobrepeso y la obesidad, al respecto reportes indican que el 34 por ciento de los niños tienen sobrepeso y obesidad, y en los últimos años ha aumentado 124 por ciento la presencia de obesidad en los niños mexicanos a su vez causada por la adquisición de hábitos alimentarios poco sanos y de actividad física deficiente ${ }^{(1)}$.

El incremento del sobrepeso y la obesidad en niños es el resultado de múltiples factores que influyen en el estilo de vida ${ }^{(3,4)}$, en este sentido estudios sugieren que el ingreso económico familiar tiene relación con el peso del hijo, es decir a menor ingreso económico, mayor probabilidad de desarrollar obesidad y por lo tanto el riesgo para el riesgo de desarrollar DM2 es superior ${ }^{(3,5)}$.

Se ha encontrado que las madres que cuentan con mayor escolaridad tienden a tener hijos con menor riesgo, en comparación a los hijos de madres con baja escolaridad quienes presentaron mayor IMC ${ }^{(6)}$.

En otros estudios se ha documentado una asociación entre el hecho de que la madre trabaje fuera del hogar lo cual aumenta el riesgo de que sus hijos desarrollen obesidad $^{(7)}$. 
Estos factores que afectan el estilo de vida se encuentran estrechamente relacionados con la parentalidad o crianza ya que afectan la alimentación y la actividad física de los niños mediante prácticas, estilos y estrategias ${ }^{(8)}$. Se sabe que las estrategias parentales de alimentación y actividad física como inculcar la actividad física y una alimentación adecuada son importantes y más en la infancia, sin embargo, se ha examinado poco sobre las estrategias parentales de alimentación y actividad física que realizan los padres a sus hijos para prevenir la DM2.

Los hábitos alimentarios y comportamientos sedentarios se adquieren en la infancia, siendo luego muy difíciles de modificar, es por ello que la influencia del ámbito familiar en el desarrollo de estos comportamientos es decisiva: los niños observan la manera de comer de sus padres, estimulan el comportamiento activo o sedentario de los hijos $^{(9)}$. Cuando los padres crean ambientes para sus hijos pueden tanto fomentar el desarrollo de comportamientos saludables como promover el sobrepeso y desórdenes alimentarios, la relación entre padres e hijos es primordial en estos aspectos. ${ }^{(10)}$

Las relaciones que los padres establecen con sus hijos tienen como resultado un conjunto de comportamientos que se pueden denominar prácticas de crianza ${ }^{(6)}$. Cuando los padres buscan influir mediante técnicas o conductas en la ingesta de alimento de sus hijos, menciona Blissett ${ }^{(12)}$ este concepto se denomina prácticas parentales de alimentación, el concepto que también puede aplicarse a la actividad física que el hijo realiza ${ }^{(13)}$.

Entre las estrategias de alimentación y la actividad física se identifican: monitoreo, control, disciplina, establecimiento de límites y reforzamiento, el monitoreo se refiere al grado en el cual los padres supervisan las conductas saludables de sus hijos, disciplina a la frecuencia con la que los sancionan por realizar conductas no saludables, control a la habilidad o el poder de los padres para hacer que sus hijos realicen lo que es deseable, establecimiento de límites a la medida en que los padres permiten a los hijos la alimentación no saludable y las conductas sedentarias, y reforzamiento al grado en que los padres elogian a sus hijos cuando realizan conductas saludables ${ }^{(13)}$.

Se ha reportado que la estrategia del control materno aumenta el riesgo de presentar obesidad en la infancia ${ }^{(14)}$, sin embargo existen otros estudios en donde mencionan que el control materno disminuye la ingesta de alimentos no saludables, aumento del consumo de alimentos saludables y reducción del peso del hijo, medido principalmente por un mayor nivel de escolaridad e ingreso económico familiar mayor $^{(15)}$.

Una serie de estrategias maternas eficaces pueden reducir el riesgo de diabetes mellitus tipo 2 en escolares. Por parte de los padres el monitoreo, la disciplina, establecer límites, tener un control y reforzamiento en la ingesta alimentaria y la actividad física se ha asociado con las prácticas de salud saludables en los niños.

A pesar de lo descrito anteriormente, en México, especialmente en Nuevo León las evidencias son insuficientes y contradictorias, motivo que guió la realización del presente estudio.

\section{Objetivos de estudio}

El propósito del estudio fue conocer las estrategias maternas de alimentación y actividad física para prevenir la DM2 y describir el peso del hijo escolar en una 
muestra de diadas madre hijo(a) en la población de Sabinas Hidalgo con el fin de saber qué estrategias realizan las madres a sus hijos escolares para prevenir dicha enfermedad y su relación con el peso del escolar.

\section{MATERIALES Y MÉTODOS}

\section{Diseño del estudio}

El diseño del estudio fue descriptivo dado que únicamente se pretende describir y documentar una situación que ocurre de manera natural ${ }^{(16)}$.

\section{Población, muestreo y muestra}

La población del estudio se conformó por 30 madres de escolares de la Escuela Primaria Niños Héroes localizada en el municipio de Sabinas Hidalgo Nuevo León, la recolección de datos se llevó a cabo en una sola fase el día 26 de Febrero del 2016. El muestreo fue aleatorio simple haciendo uso de la tabla de números aleatorios ${ }^{(17)}$.

\section{Criterios de inclusión}

1.- Madres de escolares con edades entre 6 y 12 años de edad.

2.- Niños que cursen los diferentes grados escolares.

3.- Niños que puedan mantenerse de pie por al menos 5 minutos para la toma de medidas antropométricas.

4.- Que voluntariamente y por escrito acepte participar en el estudio.

\section{Criterios de exclusión}

1.- Se excluyeron los escolares que no reunieron los criterios de inclusión y aquellos sin la autorización de los padres o tutores así como los que teniéndola manifestaron su negativa a ingresar.

\section{Procedimiento para la selección de los participantes}

Para la realización del presente estudio se contó con la autorización del Comité de Ética de la institución en la que laboran los investigadores y de la institución educativa participante donde se tuvo el contacto con las madres de familia.

Para la selección de los participantes se obtuvieron los datos de los escolares a través de las autoridades de la Escuela. Después se calculó la selección considerando los datos obtenidos, el proceso de selección de cada participante se apoyó con la tabla de números aleatorios.

Se programó una reunión informativa con las madres de familia en la institución educativa a la que pertenece su hijo el día 26 de Febrero del 2016, se les explicaron los objetivos del estudio, el propósito del estudio y los procedimientos a realizar, se solicitó la firma del consentimiento informado y se les otorgó la oportunidad de negarse si así lo deseaban. A las madres que aceptaron participar y otorgar su consentimiento por escrito, se les proporcionó y explicó el llenado del cuestionario el cual regresaron debidamente llenado el mismo día. Se les pidió llenar de manera ordenada la cedula de datos personales de la madre y del hijo y el cuestionario de 
Escala de Estrategias Parentales de Alimentación y Actividad (Parenting Strategies for Eating and Activity Scale (PEAS).

En un área especial proporcionada por la dirección de la escuela primaria se procedió a la toma de medidas antropométricas de los niños por los responsables de la investigación.

\section{Mediciones}

Se utilizó la versión en español de la escala "Parenting Strategies for Eating and Activity Scale" Estrategias Parentales para la Alimentación y la Actividad (EPAA) desarrollada por Larios (14). La escala mide las estrategias parentales sobre alimentación y actividad, cuenta con 26 reactivos, los cuales se organizan en cinco subescalas: (1) establecimiento de límites con seis ítems, (2) control con seis ítems, (3) monitoreo con siete ítems, (4) disciplina con 5 ítems, y (5) reforzamiento con dos ítems.

La escala de respuesta es tipo Lickert de 5 puntos, las subescalas de límites y de control se responden en base al grado de acuerdo, donde 1 indica desacuerdo y 5 indica acuerdo con el enunciado. Las subescalas de monitoreo, disciplina y reforzamiento, se responde en base al grado de frecuencia, donde 1 indica nunca se realiza la estrategia, y 5 indica siempre se realiza la estrategia parental.

Las puntuaciones de cada factor de la escala PEAS se convirtierten a índices de 0 a 100 y se obtienen estadísticas descriptivas. La puntuación Likert de 5 puntos se obtuvo el valor máximo 100 , en donde $100 / 5=20$, dando resultado de 20 puntos para cada respuesta, en donde $1=20,2=40,3=60,4=80,5=100$.

Cada puntuación representa la media para cada estrategia. La escala representa las estrategias enfocadas a la alimentación en 16 ítems y las estrategias sobre la actividad del niño en 10 ítems, Alimentación: Establecimiento de límites 5, 6, Monitoreo 8, 9, 10, 12, 13, Disciplina 16, 17, 18, Control 19, 20, 21, 22, 24, Reforzamiento 26, Actividad Física: Establecimiento de límites 1, 2, 3, 4, Monitoreo 7, 11, Disciplina 14, 15, Control 23, Reforzamiento 25. Se reporta validez concurrente, de constructo, contenido y predictiva. La confiabilidad reportada por subescalas varía de .73 a .87 . La escala PEAS tiene una confiabiliad alpha de Cronbach de $0.84^{(14)}$.

El peso se obtuvo con una báscula marca SECA con capacidad máxima de 200 kilogramos con una diferencia de 0.1 kilogramos, la ropa de los niños fue lo más ligera posible y se les pidió quitarse el calzado antes de subir a la báscula. La talla fue obtenida con un estadimetro de pared marca SECA con límite máximo de 2.10 metros, los niños sin zapatos y con los pies juntos de los talones, sin inclinar la cabeza hacia arriba o hacia abajo. Durante este procedimiento se tomaron medidas de seguridad.

\section{Consideraciones éticas}

El presente estudio se apegó al Reglamento de la Ley General de Salud en Materia de Investigación para la Salud. ${ }^{(18)}$. 


\section{Análisis de datos}

Los datos se analizaron con el uso del paquete estadístico Statistical Package for the Social Sciences (SPSS) versión 20, se utilizó estadística descriptiva y se recurrió al empleo de la media, desviación estándar, así como el uso de frecuencias y porcentajes, lo anterior para dar respuesta al objetivo de investigación.

\section{RESULTADOS}

Participaron 30 madres. La edad materna osciló entre 24 y 56 años, edad promedio 38.77 años $(D E=8.245)$, respecto a educación las participantes habían cursado en promedio 12.45 años $(\mathrm{DE}=0.679)$. El $50 \%$ de las madres estudiaron hasta secundaria, el $85 \%$ estaban casadas, el $76.70 \%$ se dedicaba al hogar y el $23.3 \%$ desarrollaba trabajo fuera del hogar, de éstas la mayoría desempeñaba trabajo no profesional. Refirieron un ingreso económico familiar promedio de \$6,300.00 M.N. $(D E=3,064.316)$. Respecto a los hijos, la edad osciló entre 7 y 11 años, edad promedio 9.20 años ( $D E=1.270), 53.3 \%$ del sexo masculino y el $46.7 \%$ del femenino (Tabla I)

Tabla I. Datos sociodemográficos de la madre del hijo escolar

\begin{tabular}{|c|c|c|c|}
\hline & & $f$ & $\%$ \\
\hline \multirow{5}{*}{ Escolaridad } & Primaria & 4 & 13.13 \\
\hline & Secundaria & 15 & 50.00 \\
\hline & Preparatoria & 6 & 20.00 \\
\hline & $\begin{array}{l}\text { Carrera } \\
\text { Técnica }\end{array}$ & 5 & 16.70 \\
\hline & Profesional & 0 & 0 \\
\hline \multirow{5}{*}{ Estado Civil } & Soltera & 1 & 03.30 \\
\hline & Casada & 25 & 83.30 \\
\hline & Divorciada & 2 & 06.70 \\
\hline & Viuda & 0 & 0 \\
\hline & Unión Libre & 2 & 06.70 \\
\hline \multirow[t]{2}{*}{ Ocupación } & Hogar & 23 & 76.70 \\
\hline & Empleada & 7 & 23.30 \\
\hline \multirow{3}{*}{$\begin{array}{l}\text { Número total } \\
\text { de hijos }\end{array}$} & 1 hijo & 6 & 20.00 \\
\hline & 2 a 3 hijos & 18 & 60.00 \\
\hline & 4 a 5 hijos & 6 & 20.00 \\
\hline \multirow{4}{*}{$\begin{array}{l}\text { Ingreso } \\
\text { económico } \\
\text { mensual de } \\
\text { la familia }\end{array}$} & $>5,000$ & 11 & 36.70 \\
\hline & $5,000-10,000$ & 17 & 56.70 \\
\hline & $10,001-15,000$ & 1 & 03.30 \\
\hline & $<15,000$ & 1 & 03.30 \\
\hline
\end{tabular}

Respecto al Estado Nutricio (EN) medido mediante el IMC clasificado por edad y sexo de acuerdo a lo establecido en las Cartillas Nacionales de Salud para niñas y niños de 0-9 años y Adolescentes de 10-19 años en México las cuales están basadas en lo propuesto por la OMS se identificó que un $80 \%$ de los niños presento un peso normal 
( $40 \%$ niños y $40 \%$ niñas), un $10 \%$ para sobrepeso (SP) en niños y un $10 \%$ para obesidad (OB) $\quad(6.6 \%$ niños y $3.3 \%$ niñas). La información se encuentra en la Tabla II.

Tabla II. Estado Nutricio del hijo de acuerdo al sexo

\begin{tabular}{lcccccc}
\hline Sexo & \multicolumn{2}{c}{ Niños } & \multicolumn{2}{c}{ Niñas } & \multicolumn{2}{c}{ Total } \\
IMC & $f$ & $\%$ & $f$ & $\%$ & $f$ & $\%$ \\
\hline Peso bajo & 0 & 0.0 & 0 & 0.0 & 0 & 0.0 \\
Peso normal & 12 & 40.0 & 12 & 40.0 & 24 & 80.0 \\
Sobrepeso & 3 & 10.0 & 0 & 0.0 & 3 & 10.0 \\
Obesidad & 2 & 6.6 & 1 & 3.3 & 3 & 10.0 \\
\hline Total & 17 & 56.6 & 13 & 43.3 & 30 & 100
\end{tabular}

Con respecto al nivel educativo de las madres de familia y su ocupación, se encontró que en su mayoría sus hijos demostraron peso normal, solo 6 niños demostraron sobrepeso u obesidad (Tabla III y IV).

Tabla III. Índice de Masa Corporal del hijo de acuerdo al Grado escolar de la madre

\begin{tabular}{lcccccccc}
\hline $\begin{array}{l}\text { Grado } \\
\text { escolar de la }\end{array}$ & \multicolumn{2}{c}{ Primaria } & \multicolumn{2}{c}{ Secundaria } & \multicolumn{2}{c}{ Preparatoria } & \multicolumn{2}{c}{ Carrera Técnica } \\
madre & & & & & & & & \\
IMC & $f$ & $\%$ & $f$ & $\%$ & $f$ & $\%$ & $f$ & $\%$ \\
\hline Peso bajo & 0 & 0.0 & 0 & 0.0 & 0 & 0.0 & 0 & 0.0 \\
Peso normal & 3 & 10.0 & 12 & 40.0 & 5 & 16.6 & 4 & 13.3 \\
Sobrepeso & 0 & 0.0 & 2 & 6.6 & 1 & 3.3 & 0 & 0.0 \\
Obesidad & 1 & 3.3 & 1 & 3.3 & 0 & 0.0 & 1 & 3.3 \\
\hline Total & 4 & 13.3 & 15 & 50 & 6 & 20 & 5 & 16.6
\end{tabular}

Tabla IV. Índice de Masa Corporal del hijo de acuerdo a la Ocupación de la madre

\begin{tabular}{lcccc}
\hline $\begin{array}{c}\text { Ocupación de la } \\
\quad \text { madre }\end{array}$ IMC & Hogar & \multicolumn{2}{c}{ Empleada } \\
\hline Bajo Peso & 0 & $\%$ & $f$ & $\%$ \\
Peso Normal & 18 & 59.9 & 0 & 0.0 \\
Sobrepeso & 2 & 6.6 & 1 & 20 \\
Obesidad & 3 & 10 & 0 & 3.3 \\
\hline Total & 23 & 76.6 & 7 & 0.0 \\
\hline
\end{tabular}

Respecto al objetivo de estudio, se encontró que la media más alta fue de Reforzamiento con una $\tilde{x}$ de $64.00 \quad(\mathrm{DE}=18.84)$ la cual está dirigida a elogiar a los hijos cuando realizan conductas saludables y más baja Monitoreo con 51.33 ( $\mathrm{DE}=$ 20.80) que se define como el grado en el cual los padres supervisan las conductas saludables de sus hijos. La información se muestra en la Tabla V. 
Tabla V. Estadistica descriptiva de las estrategias de la escala PEAS

\begin{tabular}{lcc}
\hline \multicolumn{1}{c}{ Estrategias } & $\tilde{x}$ & $\mathrm{DE}$ \\
\hline Limites & 63.66 & 18.84 \\
Monitoreo & 51.33 & 20.80 \\
Disciplina & 54.66 & 24.40 \\
Control & 59.33 & 19.28 \\
Reforzamiento & 64.00 & 24.80 \\
\hline
\end{tabular}

Descripción: $\quad \tilde{x}=$ Media $\quad \mathrm{DE}=$ Desviación Estándar

Respecto al IMC, al género del hijo y las estrategias maternas de alimentación y actividad física se encontró que en el caso de los niños la estrategia de Control y Limites, fueron las que demostraron medias más altas $(\tilde{x}=66.6)$ esto para quienes tenían peso normal y sobrepeso, respectivamente. En el caso de las niñas las principales estrategias que las madres llevan a cabo para prevenir DMII son Limites y Reforzamiento (71.6\% vs $75.8 \%$ ), ambas en las niñas con peso normal.

Tabla VI. Índice de Masa Corporal del hijo de acuerdo al Género y Estrategias Maternas de Alimentación y Actividad Física

\begin{tabular}{ccccccc}
\hline \multirow{2}{*}{ Sexo } & \multirow{2}{*}{ IMC } & Límites & Monitoreo & Disciplina & Control & Reforzamiento \\
& & $\tilde{x}$ & $\tilde{x}$ & $\tilde{x}$ & $\tilde{x}$ & $\tilde{x}$ \\
\hline \multirow{4}{*}{ M } & Bajo peso & 0.0 & 0.0 & 0.0 & 0.0 & 0.0 \\
& Normal & 55.8 & 48.4 & 53.4 & 66.6 & 60.0 \\
& Sobrepeso & 66.6 & 53.4 & 60.0 & 50.0 & 56.6 \\
& Obesidad & 60.0 & 40.0 & 50.0 & 45.0 & 50.0 \\
\hline \multirow{4}{*}{ F } & Bajo peso & 0.0 & 0.0 & 0.0 & 0.0 & 0.0 \\
& Normal & 71.6 & 58.4 & 58.4 & 60.0 & 75.8 \\
& Sobrepeso & 0.0 & 0.0 & 0.0 & 0.0 & 0.0 \\
& Obesidad & 60.0 & 20.0 & 20.0 & 20.0 & 20.0 \\
\hline
\end{tabular}

En la tabla VII se aprecia que las madres de familia recurren al uso de las cinco estrategias, especialmente en los niños con sobrepeso 
Tabla VII. Índice de Masa Corporal del hijo de acuerdo al Género, la Alimentación (AL) y Actividad Física (AF)

\begin{tabular}{ccccccccccccc}
\hline \multirow{2}{*}{ Sexo } & \multicolumn{1}{c}{ IMC } & \multicolumn{2}{c}{ Límites } & \multicolumn{2}{c}{ Monitoreo } & \multicolumn{2}{c}{ Disciplina } & \multicolumn{2}{c}{ Control } & \multicolumn{2}{c}{ Reforzamiento } \\
& & AL & AF & AL & AF & AL & AF & AL & AF & AL & AF \\
\hline \multirow{4}{*}{ M } & Bajo Peso & 0.0 & 0.0 & 0.0 & 0.0 & 0.0 & 0.0 & 0.0 & 0.0 & 0.0 & 0.0 \\
& Normal & 51.6 & 54.2 & 48.4 & 48.4 & 48.4 & 60.0 & 70.0 & 60.0 & 63.4 & 56.6 \\
& Sobrepeso & 60.0 & 66.6 & 53.4 & 53.4 & 53.4 & 63.4 & 53.4 & 63.4 & 60.0 & 53.4 \\
& Obesidad & 40.0 & 60.0 & 40.0 & 40.0 & 40.0 & 60.0 & 40.0 & 60.0 & 50.0 & 50.0 \\
\hline \multirow{4}{*}{$\mathrm{F}$} & Bajo Peso & 0.0 & 0.0 & 0.0 & 0.0 & 0.0 & 0.0 & 0.0 & 0.0 & 0.0 & 0.0 \\
& Normal & 66.6 & 70.8 & 58.4 & 58.4 & 56.6 & 64.2 & 65.0 & 64.2 & 76.6 & 75.0 \\
& Sobrepeso & 0.0 & 0.0 & 0.0 & 0.0 & 0.0 & 0.0 & 0.0 & 0.0 & 0.0 & 0.0 \\
& Obesidad & 60.0 & 60.0 & 20.0 & 20.0 & 20.0 & 20.0 & 20.0 & 20.0 & 20.0 & 20.0 \\
\hline
\end{tabular}

\section{DISCUSIÓN}

Se conoció las estrategias maternas de alimentación y actividad física para prevenir la diabetes mellitus tipo 2 en hijos escolares, en una muestra de 30 madres.

Al describir las Estrategias Maternas de Alimentación y Actividad física se identificó que el Reforzamiento fue la estrategia que más refirieron las madres, la cual está dirigida a elogiar a los hijos cuando realizan conductas saludables, y el valor más bajo fue Monitoreo que se define como el grado en el cual los padres supervisan las conductas saludables de sus hijos.

El haber obtenido el valor más alto en la estrategia Reforzamiento puede atribuirse a que la mayoría de las madres respondieran de manera positiva a la estrategia. Sin embargo, los autores de la escala PEAS han señalado que esta estrategia cuenta con una limitante ya que solo cuenta con dos ítems para evaluarla lo que podría ocasionar que esta estrategia no sea medida de forma adecuada o precisa ${ }^{(14,19)}$.

Las madres con nivel de estudios hasta secundaria mantienen un mejor peso en sus hijos, en cambio las madres que solo estudiaron hasta primaria tienen mayor porcentaje de hijos con obesidad.

Las madres empleadas promocionan más la salud en sus hijos, ya que un elevado porcentaje se encontró con respecto al peso normal, y las madres que se dedican al hogar fue un porcentaje menor.

Con respecto al IMC, el género del hijo y las estrategias maternas se encontró que los hijos de sexo masculino las madres refieren hacer que sus hijos realicen lo que es deseable (Control) en relación a los hijos que presentan peso normal. Y en relación a las hijas las madres refieren elogiar a sus hijas cuando realizan conductas saludables (Reforzamiento) en relación a las hijas con peso normal. Es decir, es eficaz que las madres hagan que sus hijos realicen lo que es deseable en niños, y en las niñas es eficaz que las madres las elogien cuando realizan conductas saludables.

En relación a la Alimentación en el género masculino las madres refieren hacer que sus hijos realicen lo que es deseable (Control) y en las niñas las madres refieren 
elogiar a sus hijas cuando realizan conductas saludables (Reforzamiento), esto en relación al peso normal del hijo. Respecto a la Actividad Física en los niños se encontró que las madres refieren sancionar por realizar conductas no saludables (Disciplina) y Control, en las niñas las madres refieren elogiar a sus hijas cuando realizan conductas saludables (Reforzamiento) con respecto al peso normal.

En un estudio de Flores-Peña ${ }^{(19)}$ se encontró que Reforzamiento presentó la media más alta (62.72) y Control la más baja (50.07), datos similares se encontraron en este estudio dado que según los autores de la escala PEAS esta estrategia cuenta con una limitante ya que solo cuenta con dos ítems para evaluarla, esto ocasionó que la estrategia en ambos estudios no se midiera de forma adecuada o precisa.

Lloyd (20) encontró que la estrategia Control, Establecimiento de Límites y el Reforzamiento se asociaron significativamente con la alimentación, el Reforzamiento se asoció significativamente con la actividad física infantil. En el presente estudio se encontró que las estrategias de Control y Reforzamiento obtuvieron medias más altas con respecto a la Alimentación y en Establecimiento de Límites, Monitoreo y Disciplina se encontraron medias más altas en relación a las preguntas de Actividad Física. Posiblemente las diferencias encontradas en ambos estudios están dirigidas en la zona geográfica en donde se realizó, ya que en México y Australia las madres tienen distintas costumbres y culturas y distintas estrategias enfocadas a la Alimentación y Actividad Física de sus hijos.

Ortiz-Félix ${ }^{(3)}$ en sus resultados se encontró correlación positiva y significativa en Disciplina en la alimentación y una correlación negativa y significativa en Control en alimentación. Caso contrario ocurrió en el presente estudio encontrándose que la estrategia Control obtuvo una media más alta en relación a la Alimentación y en Disciplina se encontró una media más alta en relación a Actividad Física. Lo que podría traducirse en que las madres mexicanas prefieren hacer que sus hijos realicen lo que es deseable en la Alimentación y las madres estadounidenses prefieren sancionar a sus hijos por conductas no saludables en la Alimentación, y las madres mexicanas refieren realizar esta sanción en la Actividad Física.

Al considerarse solo a las madres en este estudio podrían no arrojar resultados precisos, es importante considerar como participantes del estudio a miembros de la familia que se encarguen de los cuidados y la crianza de los hijos, esto en los casos en que la madre se encuentra fuera de casa por el trabajo y utilizar instrumentos que permitan medir los estilos de crianza, utilizando una clasificación de los estilos de crianza por ejemplo.

Los hallazgos del presente estudio de las estrategias maternas de alimentación y actividad física: Establecimiento de Límites, Control, Disciplina, Monitoreo y Reforzamiento, se encontró que las madres con hijos con sobrepeso y obesidad no permiten a los hijos la alimentación no saludable y las conductas sedentarias (Límites) y las madres con hijos con peso normal elogian a sus hijos cuando realizan conductas saludables (Reforzamiento). Otorgando un mayor límite a los hijos con sobrepeso y obesidad y otorgando mayor reforzamiento a los hijos con peso normal.

\section{CONCLUSIÓN}

Las madres elogian más a sus hijos por realizar conductas saludables y ejercen un mayor control sobre ellos para que realicen lo que es deseable con relación a la 
Actividad física y limitan, supervisan y sancionan conductas no saludables con relación a la Alimentación.

Se requieren intervenciones dirigidas principalmente a las madres para educar a ejercer las estrategias maternas de manera positiva y los riesgos que conlleva la DM2 y principalmente permitir la promoción en una alimentación adecuada y una actividad física regular y saludable con el fin de prevenir dicha enfermedad.

\section{REFERENCIAS}

1. Instituto Nacional de Salud Pública. Diabetes en adultos: urgente mejorar la atención y el control. Encuesta Nacional de Salud y Nutrición [ENSANUT]. México; 2012.

2. Instituto Nacional de Estadística y Geografía. Estadística a propósito del día mundial de la diabetes. Instituto Nacional de Estadística y Geografía [INEGI]. México; 2013.

3. Félix REO, Peña YF, Villarreal VMC, Rubia JM, Rodríguez MDR, Carranco RGH. Predictores maternos del índice de masa corporal del hijo pre-escolar y escolar. ARCHIVOS LATINOAMERICANOS DE NUTRICIÓN. 2015:65;158-165.

4. Kivimäki M, Lawlor DA, Smith GD, Elovainio M, Jokela M, Keltikangas-Järvinen L. et al. Substantial intergenerational increases in body mass indez are not explained by the fetal overnutrition hypothesis: the Cardiovascular Risk in Younf Finnss Study. Am J Clin Nutr. University College London, London, United Kingdom . 2007:86(5);1509-14.

5. Gortmaker SL, Must A, Perrin JM, Sobol AM, Dietz WH. Social and economic consequences of overweight in adolescence and young adulthood. $\mathrm{N}$ Engl $\mathrm{J}$ Med. Boston, MA, EE. UU.1993:329(14);1008-12.

6. Wells JC, Marphatia AA, Cole TJ, McCoy D. Associations of economic and gender inequality with global obesity prevalence: understanding the female excess. Soc Sci Med. London, United Kingdom. 2012:75(3);482-90.

7. Datar A, Niicosia N, Shier V. Maternal work and children's diet, activity, and obesity. Soc Sci Med. Los Angeles, Estados Unidos. 2012;196-204.

8. Ventura A, Birch L. Does parenting affect children's eating and weight status ?. Int J Behav Nutr Phys Act. 2008;5(15):5-15.

9. Verstraeten R, Van RK, Ochoa AA, Penafiel D, Holdsworth M, Donoso S, Maes L, Kolsteren P. Un marco conceptual para una alimentación saludable comportamiento en los adolescentes del Ecuador: un estudio cualitativo. PLoS One. Belgium.2014;1 (9):7.

10. Scaglioni S, Salvioni M, Galimberti C. Influence of parental attitudes in the development of children eating behavior. British Journal of Nutrition. 2008;99: 22-25.

11. Macarini SM, Martins GDF, Minetto FJ, Vieira ML. Práticas parentais: Uma revisão da literatura brasileira. Arquivos Brasileiros de Psicologia. 2010;1(62):8

12. Blissett J. Relationships between parenting style, feeding style and feeding practices and fruit and vegetable consumption in early childhood. Appetite. 2011;57:826-831.

13. Arredondo ME, Elder PJ, Ayala XG, Campbell N, Baquero B, Duerksen S. Is parenting style related to children's healthy eating and physical activity in Latino families. Health Education Research. 2006;6(21):10.

14. Larios SE, Ayala GX, Arredondo EM, Baquero B, Elder JP. Desarrollo y validación de una escala para medir estrategias de crianza latinos relacionados con comportamientos obesogénicas de los niños: Las estrategias de crianza para la Escala de alimentación y actividad (PEAS). NIH Public Access. 2009;1(10):17.

15. Pate RR, O'Neil JR, Liese AD, Janz KF, Grenberg EM, Colabiachi N. et al. Factores associated with development of excessive fatness in children and adolescents: a review of prospective studies. Obes Tev. 2013:(8):645-58. 
16. Polit DF, Hungler BF. Nursing research: Principles and methods. J.B. Lippincott Company, Philadelphia. 2004:(7).

17. Pita FS. Determinación del tamaño muestral. Unidad de Epidemiología Clínica y Bioestadística, Hospital Universitario de A Cuña. España. 2010.

18. Secretaria de Salud. Reglamento de la Ley General de Salud en Materia de Investigación para la salud. México, D.F. 1988. Recuperado el 25 de Febrero del 2016 de: www.salud.gob.mx/unidades/cdi/nom/compi/rlgsmis.html

19. Flores YP, Ortiz REF, Cárdenas VMV, Ávila HA, Alba CMA, Hernández RGC. Estrategias Maternas de Alimentación y Actividad Física y su Relación con el Estado Nutricional de los Hijos. Revista Latino-Americana.2014;22(2):86-92.

20. Lloyd BA, Lubans RD, Plotnikoff CR. Maternal and paternal parenting practices and their influence on children's adiposity, screen-time, diet and physical activity. Appetite. 2014;79:149-157. 